\title{
Difficult Airway Management in A Pediatric Case with Infantile Hemangioma
}

\author{
Abdullah Özdemir', Başar Erdivanli', Ahmet Şen', Suat Terzi', Bülent Özdemir ${ }^{3}$ \\ 'Department of Anesthesiology and Reanimation, Recep Tayyip Erdogan University School of Medicine, Rize, Turkey \\ 2Department of Otorhinolaryngology, Recep Tayyip Erdogan University School of Medicine, Rize, Turkey \\ 32Department of Neurosurgery, Recep Tayyip Erdogan University School of Medicine, Rize, Turkey
}

Cite this article as: Özdemir A, Erdivanlı B, Şen A, Terzi S, Özdemir B. Difficult Airway Management in A Pediatric Case with Infantile Hemangioma. J Emerg Med Case Rep 2018; 9: 58-60.

\begin{abstract}
Introduction: Difficult airway, one of the most fearsome situations of anesthesia practice, is a more serious problem in pediatric cases. Infantile hemangioma, the most common vascular tumour in childhood, may pose difficult airway.

Case Report: Direct laryngoscopy or videolaryngoscopy was not sufficient to secure the airway in a 7 years old child, who was diagnosed with epidural hematoma following a fall, and was scheduled for emergency surgery. She was intubated with supraglottic airway devices.
\end{abstract}

Conclusion: We aimed to present our experience in pediatric difficult airway in a case of oral infantile hemangioma.

Keywords: Infantile hemangioma, difficult airway, laringeal mask airway

Received: 17.10.2017 Accepted: 05.12.2017

\section{Introduction}

A difficult airway is a feared clinical situation for anesthetists. Particularly in children, more serious complications are likely to occur due to low reserves, different airway anatomy, and more fragile airway structures. Not enough data regarding children are available in the guidelines of the American Society of Anesthesiologists and Difficult Airway Society, whereas these guidelines state difficult airway incidence rates for adults (1). Herein, we aim to present our experience with difficult airway management in a pediatric case with hemangioma originating from the tongue base, blocking the airway by settling in the oral cavity, and complicating endotracheal intubation.

Infantile hemangioma is the most common vascular tumor in children. Although they usually regress without treatment, complications develop in approximately 10\% of cases, and surgical intervention is required (2). Depending on the size and neighboring organs of the hemangioma, life-threatening obstructions or organ failures may occur. Hemangiomas most commonly appear in the head and neck region and cause airway stenosis (3).

\section{Case Report}

A 7-year-old girl weighing $20 \mathrm{~kg}$ developed epidural hematoma due to a fall from a height, and emergency operation indication was established upon the detection of increased intracranial pressure. The past medical history provided by the family revealed a hemangioma measuring $3 \times 3 \times 1 \mathrm{~mm}$ in size beneath the tongue, for which she had undergone atrial and ventricular septal defect repair when she was 17 days old, three hemangiomas measuring $3 \times 3 \times 1$ in size on the sternum anteriorly when she was 6 months old, and one hemangioma inferior to the left knee when she was 8 months old. Therefore, steroid treatment was

\section{Address for Correspondence:}

Abdullah Özdemir, Department of Anesthesiology and Reanimation, Recep Tayyip Erdogan University School of Medicine, Rize, Turkey

E-mail: abdullah.1565@gmail.com

oCopyright 2018 by Emergency Physicians Association of Turkey - Available online at www.jemcr.org 
initiated, and she was treated after 2 months due to adrenal insufficiency. The hemangioma started to grow when she was 22 months old, and oral interferon and beta blocker were administered.

A hemangioma that originated from the tongue base, obstructing the trachea, was detected when she was 28 months old. Radiological imaging revealed that the congenital hemangioma had spread to the tongue base, lower jaw, and neck vessels, making it inoperable. Interferon, beta blocker, and thalidomide treatments were resumed. During the following 4 years, upon the growth of the hemangioma in the tongue base and upon experiencing occasional respiratory distress, high-dose steroid therapy was administered. All treatments were discontinued when gastrointestinal bleeding developed. During this process, the number of hemangiomas of the patient increased to approximately 15. Oral interferon, beta blocker, antineoplastic, and immunomodulatory treatments were initiated again. When no new hemangiomas developed following treatment, the treatment was tapered and stopped in the last 6 months. Except for a few small masses, no new hemangioma developed.

On preoperative assessment, the patient's general condition was moderate and Glasgow coma scale score was 10; she was somnolent and hemodynamically stable. Physical examination revealed a mass on the right side of the oral cavity, extending from the tongue base to the trachea and blocking approximately half of the airway (Figure 1). Cranial computerized tomography performed when she was brought to the emergency room after the fall demonstrated that the mass started from the hyoid bone level and extended superiorly, constricted the airway by covering a major part of the vallecula and tongue base, and pushed the airway to the left (Figure 2). The Ear, Nose and Throat Clinic was informed in case of a potential emergency tracheotomy. Preparation for difficult airway management was done with video laryngoscopy, fiberoptic bronchoscopy, and laryngeal masks with fit size.

The pre-induction mask ventilation of the patient taken in the operating room was comfort. Following the induction of general anesthesia, muscle relaxation was provided with rocuronium. Direct laryngoscopy was performed by avoiding compression on the hemangioma; however, the epiglottis behind the mass could not be visualized. The trachea could be entered with an adult-sized fiberoptic bronchoscope, but the endotracheal tubes delivered through the adult-sized fiberoptic bronchoscope were larger than the patient's trachea. Intubation was attempted with video laryngoscope, but the blade could not be placed due to the mass.

Thereupon, tracheal intubation was abandoned, and ventilation was provided by placing the laryngeal mask paramedially so that it would lean against the left side. No problem occurred during oxygenation and maintenance of inhaler anesthesia. The hemodynamically stable patient was decurarized and awakened with sugammadex after approximately 45 minutes when the surgery was completed. The patient, who was kept in the recovery room for 2 hours, was transferred to the neurosurgery clinic with an Aldrete score of 10 upon not observing any complication. She was discharged on postopera-

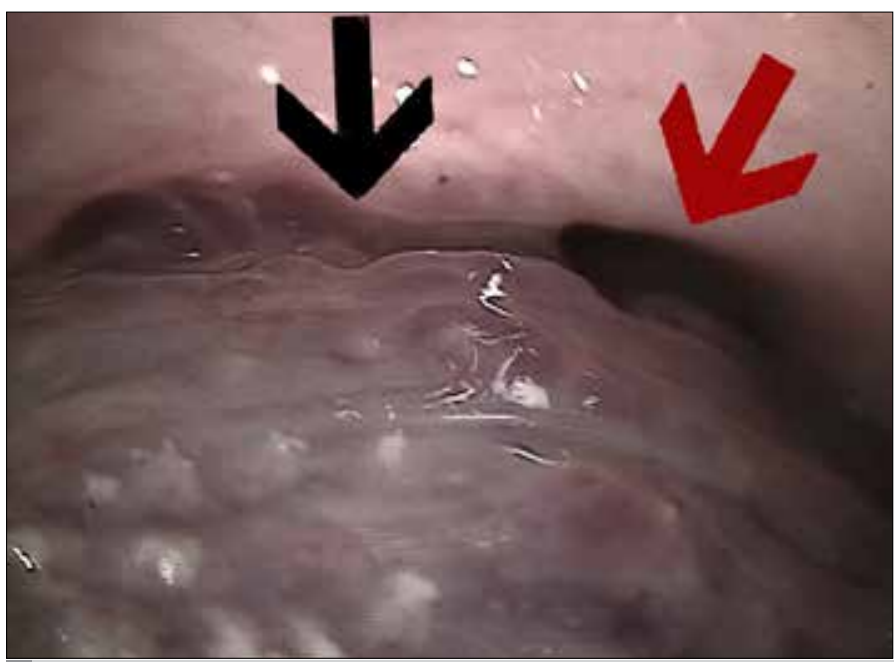

FIGURE 1. Video laryngoscope. Image of the mass filling the oropharynx and obstructing the airway (black arrow: mass, red arrow: airway).
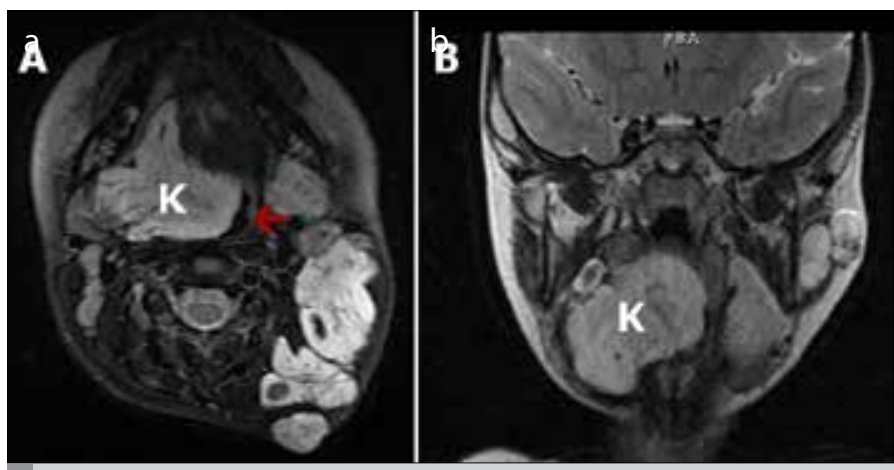

FIGURE 2. a, b. Computerized tomography. Image of the mass in the oropharynx and airway (a) in axial section (K: mass, red arrow: airway); and (b) in coronal section depicting the tongue base and airway.

tive day 2 and was started to be followed up with the control visits in the outpatient clinic.

\section{Discussion}

A difficult airway is a problematic condition even in adult patients and under elective conditions. The difficulty increases further under shift work conditions and in pediatric patients. Because it is easier to establish the emotional and hemodynamic status of patients; to provide preparations for anesthesia; and to provide assisting staff, equipment, and the support of other clinics and assisting anesthesiologists under elective conditions. It was a major disadvantage that our patient underwent operation under emergency and night conditions. The localization of the mass and openness of airway were re-evaluated with the computerized tomography obtained due to trauma.

Although the number of intubation attempts for children in the difficult airway guidelines is limited to 3-4 times, we delimitated the laryngoscopy procedure with two trials due to the possibility 
of bleeding associated with laryngoscopy from the hemangioma within the oral cavity (4).

Hemangiomas can cause various complications, ranging from cosmetic problems to airway obstruction depending on their localization. They may appear in the eyes, lips, ears, and around the neck. In case of enlargement, they may lead to complications such as liver failure, heart failure, or ulceration (2).

Hemangiomas comprise $1.5 \%$ of all laryngeal anomalies (5). In our case, the mass measuring $2 \times 3 \mathrm{~cm}$ in size was located in the supraglottic area. No newly developed hemangioma was observed in our patient who had received recent treatment. There was no complication other than serious obstruction in the airway.

In recent years, supraglottic airway devices have varied in terms of both shape and flexibility of material, and they have strengthened the anesthetist's hand as an alternative to invasive devices in a difficult airway (6). In a study conducted in the UK on the use of supraglottic devices in pediatric patients, nearly all of the participants reported that they consider supraglottic devices as a significant advantage in a difficult airway (7).

In addition, another study on patients with osteogenesis imperfecta showed that laryngeal mask airway and total intravenous anesthesia can be used safely (8). Although laryngeal masks are not considered safe for surgical procedures that require prone and lateral positions in neuroanesthesia, they can be used in the supine position for appropriate cases. Additionally, the classical type that we placed paramedially in our patient was not appropriate for intubation, whereas tracheal tubes can be placed through the second generation of these devices.

Considering the difficult airway algorithm, if the need for surgery remains in case of a failed intubation procedure after second-line attempts, it was stated that supraglottic devices and difficult mask ventilation algorithm should be used (4). In addition, although there was the option of fiberoptic intubation through a laryngeal mask, we decided to resume surgery because safe and adequate ventilation could be provided with the laryngeal mask in our case.
With our case, we tried to emphasize that based on the difficult airway algorithm, a complete multidisciplinary coordination should be conducted with necessary specialty fields in addition to preparing case starting from the preoperative period, with all airway devices and tools available for potential complications.

Informed Consent: Written informed consent was obtained from patients parents who participated in this study.

Peer-review: Externally peer-reviewed

Author Contributions: Concept - A.Ö., A.Ş.; Design - A.Ö., S.T.; Supervision B.Ö., B.E, A.Ö.; Resources - A.Ö., S.T.; Materials - A.Ö., S.T.; Data Collection and/ or Processing - A.Ö., A.S.; Analysis and/or Interpretation - A.Ö., S.T., B.E; Literature Search - A.Ş., A.Ö.; Writing Manuscript - A.Ö., B.Ö.; Critical Review - A.Ş., B.E., S.T.

Conflict of Interest: The authors have no conflict of interest to declare.

Financial Disclosure: The authors declared that this study has received no financial support.

\section{References}

1. Tong DC, Beus J, Litman RS. The Children's Hospital of Philadelphia difficult entubation registry. Anesthesiology 2007; 107: A1637.

2. Cheng CE, Friedlander SF. Infantile hemangiomas, complications and treatments. Semin Cutan Med Surg 2016; 35: 108-16. [CrossRef]

3. Balakrishnan K, Perkins JA. Management of airway hemangiomas. Expert Rev Respir Med 2010; 4: 455-62. [CrossRef]

4. Difficult airline guide for children aged 1-8. Address: www.tard.org.tr/ assets/kilavuz/10.pdf, accessed on 15.10.2017.

5. Ahmad SM, Soliman AM. Congenital anomalies of the larynx. Otolaryngol Clin North Am 2007; 40: 177-91. [CrossRef]

6. Uzun \$̧, Şahin A, Köse EA, Aypar Ü. Fiberoptic Intubation via lariyngeal mask airway in patient with unexpected difficult airway. Türkiye Klinikleri J Anest Reanim 2008; 6: 28-32.

7. Bradley AE, White MC, Engelhardt T, Bayley G, Beringer RM. Current UK practice of pediatric supraglottic airway devices - A survey of members of the Association of Paediatric Anaesthetists of Great Britain and Ireland. Paediatr Anaesth 2013; 23: 1006-9. [CrossRef]

8. Erdogan MA, Sanli M, Ersoy MO. Anesthesia management in a child with osteogenesis imperfecta and epidural hemorrhage. Braz J Anesthesiol 2013; 63: 366-8. [CrossRef] 